\title{
EL DISEÑO DE UN ESTADO DEMOCRÁTICO FEDERAL VIABLE*
}

\author{
PETER C. ORDESHOOK \\ Californian Technological Institute
}

\author{
PALABRAS CLAVE ADICIONALES ADDITIONAL KEYWORDS \\ Federalismo, Confederación, Constitución, Federalism, Confederation, Constitution, \\ Alemania, EEUU, Canadá, Rusia. \\ Germany, Russia, USA, Canada.
}

\begin{abstract}
RESUMEN. El objetivo de este artículo es analizar cuáles son las condiciones necesarias para diseñar un Estado democrático federal integrado y viable. Una de estas condiciones se refiere al sistema de partidos. Se requiere un sistema de partidos que propicie que los lideres locales o regionales sean parte del gobierno nacional. Para ello, lo más adecuado son partidos nacionales con una fuerte base regional. La segunda condición es una presidencia débil, que no pueda gobernar directamente sin contar con los líderes políticos regionales; tendrá que emplear el potencial integrador de su partido mediante el uso de lo que generalmente se denomina "liderazgo".
\end{abstract}

\begin{abstract}
The aim of this paper is to analyse the necessary conditions for the design of an integrated and viable democratic federal state. The first requisite concerns the character of the party system. A federal party system affords local and state leaders the potential opportunity to ascend to national-level office. Accordingly, national parties with strong regional bases are ideal. The second requisite is a constitutionally weak chief executive who would be unable to govern directly without taking into account regional leaders. The president would have to employ his party's integrating potential, through the use of what is generally called "leadership".
\end{abstract}

\section{E-mail: ordeshook@hss.caltech.edu}

"Esta investigación fue financiada por una beca del Consejo Nacional para la Investigación Soviética y del Este de Europa del Instituto de Tecnología de California. El autor desea además agradecer a Mijail Filippov sus muchas sugerencias provechosas en la preparación del manuscrito.

Traducción de Francisco Herreros Vázquez.

Revista Internacional de Sociología (RIS)

Tercera Época, n 32, Mayo-Agosto, 2002, pp. 201-220. 
RIS

REVISTA INTERNACIONAL DE SOCTOLOGIA

№ 32, Mayo-Agosto, 2002

PETER C. ORDESHOOK

\section{INTRODUCCIÓN}

Alexis De Tocqueville escribió que la "constitución de los EEUU es un trabajo admirable. Sin embargo, se puede considerar que sus fundadores no habrían tenido éxito si los 150 años previos no hubieran proporcionado a los diferentes Estados de la Unión el gusto y la práctica del gobierno provincial". No hay duda de que América se benefició del hecho de que sus cámaras legislativas coloniales - la personificación legal de la oposición a Gran Bretaña y a los gobernadores nombrados por la Corona - fueran fácilmente transformadas en organismos gubernamentales y en asambleas constituyentes para elaborar proyectos de constituciones estatales. La experiencia con esas constituciones, a su vez, se demostró inestimable a la hora de redactar el proyecto del documento nacional. Y, en general, América se benefició del hecho de que nació con una dotación casi completa de sistemas electorales regionales, facciones regionales que sirvieron como semilleros para los partidos estatales y nacionales, tribunales regionales, burocracias públicas regionales $y$, por muy limitado que fuera el derecho al voto, un electorado familiarizado con cambios de poder impuestos por el voto popular.

Por desgracia, puesto que la experiencia americana de gobernarse a sí misma, y en particular el federalismo, dio comienzo con muchos de los componentes esenciales de una democracia plena, se cree por lo común que las lecciones aprendidas de este experimento son irrelevantes para otras democracias más nuevas, especialmente aquéllas que están realizando hoy en día la transición de un gobierno autoritario a otro democrático. Por el contrario, los que intentan dirigir o influir en esas transiciones sostienen que Estados tales como España o Taiwan, con similares predecesores autoritarios, ofrecen las lecciones o modelos más relevantes. Nosotros, sin embargo, estamos en desacuerdo. Puede ser verdad que tales casos ilustren mejor los principios generales de una transición. Pero las democracias maduras como los EEUU ilustran mejor los principios de unas relaciones federales estables y el diseño apropiado de aquellas instituciones democráticas que deben ser diseñadas con un lenguaje constitucional cuando comienza el proceso de transición. Las transiciones contemporáneas que han tenido éxito podrían ilustrar mejor el "cómo llegar allí, pero las democracias estables ilustran mejor lo que hay "alli".

De hecho, en De Tocqueville hallamos un problema diferente. Concretamente, se le interprete como se le interprete, las condiciones a las que él se refería no pueden ser ni necesarias ni suficientes para la creación de un Estado democrático estable, federal o de otro tipo. No pueden ser suficientes, ya que las mismas circunstancias describen la Europa de hoy, aunque su integración política sigue siendo fundamentalmente una promesa. Describen no sólo la América de 1787, sino también la de 1860, anterior al estallido de su Guerra Civil, y describen al Canadá contemporáneo a pesar de la amenaza de secesión de Quebec. No pueden ser necesarias, ya que sólo la interpretación más liberal de las mismas nos per- 
mite decir que describen a Alemania después de la Segunda Guerra Mundial, a España después de Franco, o a India después de la independencia. No obstante, De Tocqueville es universalmente relevante ya que sus observaciones exigen que nosotros entendamos en términos teóricos generales la naturaleza exacta de las ventajas que América obtuvo de sus experiencias coloniales y preconstitucionales. Aunque seamos incapaces de reproducir las condiciones de que disfrutó América en 1787, no deberíamos suponer que esas condiciones no son nada más que una forma concreta de satisfacer ciertas condiciones generales necesarias y suficientes para un federalismo estable.

Este ensayo intenta identificar esas condiciones. Nuestro objetivo es determinar si redactar constituciones para una nueva democracia simplemente eligiendo aquellas características institucionales empleadas por otros Estados que parecen más adecuadas para lograr la estabilidad en sus correspondientes entornos, pero sin el beneficio de una buena teoría, es más o menos probable que genere una configuración institucional general que frustre la realización de los objetivos buscados. Estamos especialmente interesados en el hecho de que la mayoría de los comentarios sobre federalismo se centran en cosas distintas de aquéllas que afectan a lo que aquí sostenemos que es, como mínimo, una condición necesaria para la estabilidad, a saber, el autocumplimiento del acuerdo federal y el papel de los partidos políticos en ese autocumplimiento'. Y nuestro interés se ve incrementado por el hecho de que aun cuando se consideran los parámetros institucionales críticos, existe una inclinación natural a realizar precisamente las elecciones erróneas. Primero, porque al suponer por lo común que los ciudadanos en las nuevas democracias son votantes no cualificados, fácilmente confundidos por apelaciones nacionalistas o étnicas, se piensa que pocos cargos públicos deberían ser ocupados inicialmente por elección directa. En segundo lugar, debido a que se supone o bien que los gobiernos regionales son corruptos o demasiado fácilmente corruptibles y a que las elecciones regionales son vistas como fáciles

\footnotetext{
'Dos notables excepciones a esta generalización son el ensayo de Riker (1964) y el estudio general de partidos en Estados federales de Chandler (1987). Frente a ello, véanse los volúmenes compilados por Tushnet (1990); Burgess y Gagnon (1993); Smith (1995); Ostrom (1991), que considera sólo la representación en el parlamento nacional, y el capitulo dedicado al federalismo en el texto de Lijphart (1984), que no menciona ni a los partidos ni a las elecciones. Ningún ensayo del número 25: 2, Primavera de 1995 de la revista Publius, que celebraba su 25 aniversario de publicaciones sobre federalismo, cita el papel de las elecciones y de los partidos. El estudio de regulaciones constitucionales ofrecidas allí (Saunders, 1995: 61-80), que están pensadas para ser más pertinentes para el diseño federal, se centran en cambio en el papel de los sujetos federales al reformar la constitución nacional, la autoridad del poder judicial, los acuerdos de tratados, la asignación de responsabilidades políticas, las garantías de los mercados internos, el desarrollo económico, la autoridad para regular impuestos, la disparidad de ingresos regionales, y la estructura y autoridad de la legislatura nacional, poniendo el énfasis en la representación del sujeto federal.
} 
blancos para el fraude, las elecciones regionales y locales son aplazadas hasta que se alcance un determinado grado de estabilidad política a nivel nacional, o bien son sometidas a una estricta vigilancia y control por parte del gobierno. En tercer lugar, debido a que demasiada gente se adhiere a una visión ingenua de la democracia según la cual las elecciones se consideran mecanismos para medir o determinar la "voluntad popular", las elecciones para el parlamento nacional, para los gobiernos regionales y locales y para presidente, tienen lugar por lo común en momentos diferentes, en la creencia de que haciéndolo así se evita confusión en la expresión de esa voluntad. En cuarto lugar, puesto que el jefe del gobierno (el presidente) es comúnmente identificado como la cabeza del orden constitucional del Estado, se supone que los contendientes para ese cargo deberían estar "por encima de los partidos" o que, una vez elegidos, no deberían ostentar ningún cargo oficial en un partido. Finalmente, puesto que la mayoría de los Estados que están realizando la transición simultánea de la planificación estatal centralizada hacia una economía de mercado y de un gobierno autoritario a una democracia, se supone que necesitan una política nacional coherente, se piensa que los jefes de gobierno, especialmente en regímenes presidenciales, tienen que tener mucho poder, incluyendo la autoridad para legislar cuando no lo hace el parlamento nacional. Nosotros, sin embargo, sostenemos que cada una de estas decisiones es la errónea para diseñar un Estado federal estable.

Antes de seguir adelante, hay que señalar dos cosas. En primer lugar, nuestro énfasis en el federalismo no significa que consideremos las formas federales necesariamente superiores a las unitarias. Los Estados pequeños, especialmente los culturalmente homogéneos que tienen que competir económicamente con sus vecinos grandes, pueden tener poco que ganar con el federalismo. Asímismo, alguna forma de autonomía regional parece ineludible en cualquier Estado "razonablemente" grande que debe lidiar con divisiones étnicas, religiosas o lingüísticas con una base geográfica (Horowitz, 1985 y 1991) o que pudiese encontrar irresistible adoptar políticas proteccionistas (Buchanan, 1995). Sin embargo, en lugar de revisar los argumentos en favor del federalismo, suponemos que se ha tomado la decisión de adoptar una forma federal. En segundo lugar, consideramos que muchas de las cosas que podrían facilitar un proceso democrático estable no están siempre sujetas a manipulación. Nos referimos aquí a esas cosas imprecisas a las que se etiqueta comúnmente como "cultura", así como a los asuntos frecuentemente olvidados de geografia política. Sin dejar a un lado la hipótesis de que tales cosas son profundamente importantes a la hora de determinar el carácter último del Estado, nuestra atención se centra en la posibilidad de manipular cosas que son el producto consciente del diseño humano (instituciones políticas tales como los modos de representación, la estructura y el momento de las elecciones, y la autoridad constitucional de las ramas ejecutivas y legislativas del gobierno). La cultura y la geografía pueden influir en cómo configuraciones institucionales específicas actúan de forma que establecen expectativas sobre dicha actuación que 
se autocumplen, pero aún debemos identificar en términos teóricos generales los principios fundamentales del diseño que guían nuestra elección de un conjunto de instituciones frente a otro para una sociedad específica.

\section{CONFUSIONES}

Cuando diseñamos una federación democrática, el primer problema con el que nos encontramos es de definición: ¿Qué es un estado federal? ¿Debe ser identificado explícitamente como tal en su constitución? ¿Debe adoptar el gobierno nacional una forma particular (por ejemplo, una cámara legislativa superior con autoridad significativa y una explícita representación regional)? ¿Qué papel desempeña la palabra "soberanía" frente a las partes integrantes de la federación? ¿Debería haber definiciones distintas para palabras tales como "federal", "federalismo", "federación" y "confederación"?.

$\mathrm{El}$ que las respuestas satisfactorias a estas preguntas, universalmente reconocidas como tales, se nos escapen, sugiere que discutir sobre tales cosas no es una forma especialmente productiva de proceder. Sin embargo, obsérvese que las respuestas que comúnmente ofrece la gente, relacionadas con palabras como "autonomía", "supremacía", "secesión", "representación" y "soberanía", nos obligan a pensar en las federaciones y en las relaciones federales en términos de cierta interacción participativa de tipo $N+1$ entre los $N$ sujetos federales y el gobierno nacional. Aunque sostengamos en breve que diseñar una federación sobre la base de tal conceptualización sólo puede producir un diseño imperfecto, tal conceptualizapción es, no obstante, valiosa, pues nos obliga a confrontar dos cuestiones clave. En primer lugar, nos obliga a preguntarnos cómo se deberían asignar las jurisdicciones políticas entre gobiernos, quién debería vigilar servicios públicos tales como la educación y el transporte público, cuáles deberían ser las competencias en materia fiscal de los gobiernos regionales, cuál el papel del gobierno nacional en la regulación de las elecciones regionales, cuáles los métodos para designar o elegir a los poderes ejecutivos regionales y qué estructura global de la judicatura nacional y regional. En segundo lugar, tal conceptualización requiere que entendamos cómo pueden permanecer estables algunas asignaciones, cómo pueden afrontar los asuntos no previstos, y cómo puede mantener un Estado el "contrato federal" por el cual ningún gobierno sobrepase sus límites, por el cual la secesión no amenace la viabilidad del Estado, y por el cual toda renegociación del acuerdo federal se produzca sólo a través de medios democráticos y constitucionales "naturales".

Lo destacado de estas dos cuestiones se deriva de cualquier conceptualización que plantee una relación entre centro y periferia dominada, incluso sin divisiones étnicas y lingüísticas, para asuntos tales como la competencia por los ingresos fiscales, el control de recursos naturales y las inversiones. Sin embargo, aunque una 
RIS

REVISTA INTERNACIONAL DE SOCIOLOGIA

No 32, Mayo-Agosto, 2002

PETER C. ORDESHOOK

conceptualización del federalismo como $N+1$ participantes nos fuerce a confrontar estos dos asuntos, ninguna teoría del federalismo basada exclusivamente en ella puede proporcionarnos una buena guía cuando diseñamos instituciones democráticas federales. En cualquier modelo político puramente de $N+1$ participantes en el cual la autonomía de los jugadores, si no la constitución nacional misma, esté sujeta continuamente a renegociación, el único mecanismo por el cual se puede defender la autonomía, aparte de retirarse del juego (secesión), implica algún tipo de proceso de formación de coaliciones potencialmente divisor o desestabilizador. Y aquí las dificultades surgen casi inmediatamente. El gobierno central de todo Estado ha de ser autorizado a regular las relaciones interregionales y a asegurar el suministro eficiente de aquellos servicios públicos que se le asignan "normalmente". Pero la autoridad para hacer tales cosas, junto con la regulación constitucional habitual que otorga supremacía a la ley federal, amenaza necesariamente la autonomía de los gobiernos regionales. Si concluimos, entonces, que la protección primordial de los sujetos federales contra el centro es una coalición "ganadora" de los sujetos federales contra el centro, no sabemos todavía por qué el centro no puede formar una coalición que trabaje para crear desventajas a las regiones que no estén aliadas con él. Y aunque la representación explícita en el parlamento nacional convierte a los sujetos federales en una parte del gobierno nacional, no podemos excluir coaliciones de regiones contra otras regiones o la parálisis total del Estado que podría llevar a algunos sujetos federales a preferir la secesión.

Nuestro argumento, entonces, es que si al considerar nuestra conceptualización del federalismo democrático no logramos ir más allá de una caracterización del asunto como de $N+1$ participantes y no consideramos el sistema federal como un todo, políticas intraparticipantes incluidas, es poco probable que creemos instituciones públicas que aseguren relaciones federales estables. Considérese, por ejemplo, el supuesto de que unas relaciones federales estables puedan ser aseguradas mediante una cuidadosa enumeración constitucional de la autoridad de los gobiernos nacionales y regionales o, como en Rusia, mediante tratados entre esos gobiernos. El problema en ese caso es que, en ausencia de otras protecciones institucionales, probablemente ningún acuerdo estará libre de posteriores demandas de renegociación. De hecho, al describir los Estados Unidos, Woodrow Wilson (1911: 173) afirmó que la "cuestión de la relación de los Estados con el gobierno federal es la cuestión cardinal de nuestro sistema constitucional. Cada encrucijada de nuestro desarrollo nacional nos ha situado cara a cara frente a esa cuestión y ninguna definición de los hombres de estado ni de los jueces la ha acallado o decidido. De hecho, no puede ser resuelta por una generación, pues es una cuestión de crecimiento, y cada estadio sucesivo de nuestro desarrollo político y económico le da un nuevo aspecto, hace de ella una cuestión nueva". Puesto que no tenemos razón para creer que las cosas serían diferentes en cualquier otro Estado, debemos suponer que tal renegociación es parte inherente de todo sistema 
federal, lo cual, si es cierto, supondrá en el mejor de los casos que los tratados y la asignación constitucional de jurisdicciones políticas sean meros expedientes temporales. Y son expedientes peligrosos si, por centrarnos en ellos, ignoramos las cosas que dicta el curso de su renegociación.

Frente a lo anterior, considérese la opinión de que la prosperidad económica es la clave de la estabilidad política. El argumento en este caso es que si los beneficios de la federación son lo suficientemente grandes y la disolución de la federación es algo demasiado costoso, entonces la estabilidad está asegurada ${ }^{2}$. Este argumento tiene, sin embargo, dos problemas. En primer lugar, ignora el hecho de que la mera prosperidad no tiene por qué afectar a los incentivos de los decisores individuales en su búsqueda de ventajas a costa de otros. Mientras los incentivos conflictivos de la gente les induzcan a la no cooperación, seguirán sin cooperar con independencia de su grado de prosperidad. Lo que queremos decir resulta fácilmente ilustrado por el juego, ampliamente discutido, del dilema del prisionero, que se recoge en la tabla 1 , en el cual a cada persona se le ofrecen dos elecciones: cooperar (C) o no cooperar (NC). Si ambos eligen $\mathrm{C}$, entonces ambos reciben un pago de 5 , mientras que si ambos eligen $\mathrm{NC}$, ninguno obtiene nada (el primer número de cada celda es el pago del jugador de fila; el segundo, el del jugador de columna). El dilema aquí consiste en que incluso si ambos están de acuerdo en elegir $\mathrm{C}$, cada uno tiene un incentivo para desertar unilateralmente a $\mathrm{NC}$, con independencia de que crean o no que el otro acatará algún tipo de acuerdo. Dicho esto, para representar el incremento en la prosperidad potencial derivado de la cooperación, supóngase que multiplicamos todos los pagos positivos por 100 para que la cooperación produzca a cada uno un beneficio de $500^{3}$. Pero obsérvese que esto no altera los incentivos básicos de la situación. Cada jugador preferirá todavía elegir NC, con independencia de lo que elija su oponente. Obsérvese, por otra parte, que aunque multiplicar -6 por 100 parece dar a la gente un incentivo mayor para resolver su dilema, suponer que se resolverá sólo nos lleva a plantear la cuestión desde el punto de vista del mecanismo que se supone que afecta a la solución y, por lo tanto, no contradice la opinión de que el mero hecho de poner en peligro la prosperidad es insuficiente para asegurar la cooperación.

\footnotetext{
${ }^{2}$ En relación con la adopción implícita y a veces explícita de esta opinión en el contexto del ofrecimiento a Rusia de prescripciones políticas, véase Wallich (1994).

${ }^{3}$ Por supuesto, se podría sostener que la prosperidad incrementaría ciertos pagos —en especial los que corresponden a la casilla $(\mathrm{C}, \mathrm{C})$ - más que otros, de forma que el juego ya no sería un dilema del prisionero. Sin embargo, el argumento exige dar cuenta de forma más detallada no sólo de la estructura de las relaciones económicas, sino también de las políticas, pues ahora no es sólo la prosperidad per se lo que importa, sino la naturaleza precisa de su dependencia de la cooperación.
} 
RIS

REVISTA INTERNACIONAL DE SOCIOLOCIIA

№ 32, Mayo-Agosto, 2002

PETER C. ORDESHOOK

Tabla 1.

\begin{tabular}{rcc}
\hline & $\mathrm{C}$ & $\mathrm{NC}$ \\
\hline $\mathrm{C}$ & 5,5 & $-6,6$ \\
$\mathrm{NC}$ & $6,-6$ & 0,0 \\
\hline
\end{tabular}

Aún queda disponible, sin embargo, una solución potencial de estos dilemas para aquéllos que abogan por la centralidad de los asuntos económicos: el hecho de que tales dilemas se pueden resolver si el juego se repite indefinidamente. La repetición permite a los jugadores castigar la deserción de cualquier acuerdo cooperativo, y la deserción no ocurrirá si todos los jugadores otorgan al futuro suficiente importancia ${ }^{4}$. Se podría argumentar, entonces, que la prosperidad económica da confianza a los jugadores de que el juego se repetirá e incrementa sus incentivos para asegurar de algún modo la repetición. Pero en este caso nos tropezamos con dos problemas subsidiarios. El primero es que la hipótesis económica evita la cuestión de la simultaneidad: ¿es la prosperidad una garantía de la estabilidad o es la estabilidad la que asegura la prosperidad? Si la relación causal más importante es la segunda, entonces los simples intentos de asegurar la estabilidad centrándose en políticas económicas llevarán con mayor probabilidad a resultados no previstos o completamente insatisfactorios. El segundo problema, que quizás sea más importante, es que si los jugadores llegan a creer que existe una posibilidad razonable de que el juego no se repita indefinidamente, la cooperación finaliza, y su creencia se convierte en una profecía que se autocumple. En otras palabras, basar la estabilidad en la premisa de la prosperidad económica hace poco más que crear una federación especialmente vulnerable a los caprichos de la política económica y socava una de las razones principales para construir un Estado: asegurar la estabilidad política y social en circunstancias económicas imprevistas y potencialmente turbulentas.

Quizás no debería sorprendernos, entonces, que los padres de la Constitución de los EEUU, dado su conocimiento de la ciencia política, no confiaran en las promesas de prosperidad para lograr la viabilidad de su confederación. De hecho, se interesaron más bien por una relación que operaba en dirección opuesta: los acuerdos institucionales configurados bajo los artículos de la Confederación que

${ }^{4}$ Para la formulación matemática precisa de esta discusión véanse, por ejemplo, Taylor (1976); Friedman (1977), o cualquier libro de texto de teoría de juegos. 
amenazaban la prosperidad. En realidad, Madison y Hamilton, al menos, parecen haber rechazado la conceptualización de $N+1$ participantes como base para la construcción de la república americana. Puede que sus palabras fueran simple retórica, pero en su opinión, una federación estable no puede ser meramente un Estado próspero en el cual los sujetos federales nos son sino otro conjunto de intereses que debe competir con el gobierno nacional por poder e influencia. En lugar de ello, las identidades de los $N+1$ participantes deben ser eclipsadas de algún modo, y una constitución federal debe "convertir [a los gobiernos de los Estados] en partes integrantes de la soberanía nacional" (El Federalista $\mathrm{n}^{\circ}$ 9).

No podemos estar seguros de lo que Hamilton y Madison querían decir con "parte integrante", aunque sabemos que los mecanismos constitucionales de supremacía, representación, estructura bicameral del parlamento nacional, separación de poderes en todos los niveles de gobierno, acceso directo para el electorado a todos esos niveles, y control regional de los cargos regionales fueron considerados necesarios para este fin. Pero como deja claro El Federalista con su énfasis repetido en el propio interés individual y el papel que las instituciones desempeñan al dirigir ese interés, esas instituciones fueron meros mecanismos diseñados para satisfacer una condición más general. Lo que sugerimos es que en su forma más básica, esa condición, que podemos considerar como la característica definitoria de una federación integrada, es la siguiente: sostener un acuerdo federal que "deje [a los sujetos federales] en posesión de ciertas parcelas de poder soberano exclusivas y muy importantes" ( $\mathrm{El} \mathrm{Federalista} \mathrm{n}^{\circ}$ 9) requiere acuerdos institucionales que generen incentivos para que las élites políticas regionales cooperen y se coordinen entre sí y con el gobierno nacional, y que generen incentivos para que las élites nacionales, incluyendo a aquellas no específicamente pensadas para representar a los gobiernos regionales, protejan la autonomía de todos los sujetos federales. Esos intereses para cooperar, coordinar y proteger, deben ser, además, transparentes y evidentes para todo el mundo.

\section{PARTIDOS Y ELECCIONES}

El término clave en nuestra caracterización de una federación integrada es el "interés propio", ya que el propio interés individual es la única forma de hacer que un contrato federal se cumpla por sí mismo. Pero lo que debemos hacer ahora para conseguir que nuestra caracterización sea algo más que una simple prescripción utópica es especificar los acuerdos institucionales más probables para generar los motivos deseados. Para ese fin, ya que no tenemos interés en construir una federación autoritaria, comenzamos con las características que definen una democracia: el hecho de que las élites políticas (con la excepción quizás del poder judicial) deben ganar elecciones para asegurar y mantener los cargos. Mientras que la gente puede buscar un cargo público por las más diversas razones - aceptación 
RIS

REVISTA INTERNACIONAL DE SOCIOLOGIA

NN 32, Mayo-Agosto, 2002

pública, hacer el "bien", o por beneficio personal - no pueden llevar a cabo ninguna de estas cosas si no consiguen ganar las elecciones. Se sigue, entonces, que la autoridad de los cargos públicos electos, las reglas para seleccionar a estos cargos (v.g., la definición de los vencedores y los procedimientos de nominación del partido), y el papel de los partidos a la hora de facilitar la elección y reelección de los candidatos, en combinación con las preferencias de los votantes, son las claves que determinan el interés propio de un cargo electo.

Combinar este hecho con nuestro primer principio implica que la estructura general del sistema electoral de un Estado es un componente crítico del diseño institucional federal.

En breve desarrollaremos esta implicación y la usaremos para derivar algunos consejos prácticos sobre el diseño institucional. Sin embargo, antes de confiar en un argumento puramente teórico, es provechoso considerar una ilustración sustantiva específica. Y aquí lo más revelador es considerar la experiencia de EEUU durante lo que se puede considerar como el fracaso más grande del federalismo, la Guerra Civil. Brevemente, siguiendo el análisis de aquella época de McKitrick (1967) podemos comenzar observando que tanto la Confederación como la Unión operaron bajo sistemas constitucionales prácticamente idénticos $\mathrm{y}$, al menos cuando comenzó la guerra, ni el Norte ni el Sur se diferenciaban radicalmente en términos de prosperidad económica global (Easterlin, 1960). Sin embargo, a pesar de estas semejanzas, Lincoln tuvo mucho mayor éxito en la coordinación del esfuerzo de la Unión en la guerra que el que tuvo su homólogo Confederado, Jefferson Davis. Lincoln tuvo éxito, por ejemplo, en asegurar el control nacional de las milicias estatales, mientras que Davis se tropezó en general con los gobernadores de los distintos Estados, que se negaron a cooperar incluso en momentos decisivos, cuando el Sur parecía tener ventaja militar. Ya que las circunstancias económicas iniciales y las estructuras políticas constitucionales fueron casi equivalentes, los historiadores se centran normalmente en las características personales de Lincoln y Davis cuando intentan explicar las diferencias en el liderazgo. McKitric, sin embargo, considera una hipótesis diferente, a saber, la existencia de un sistema de partidos competitivo en el Norte y un sistema no competitivo en el Sur. Tras la secesión, el Norte no sólo retuvo al partido Republicano, sino al ala norteña del partido Demócrata. El Sur, en contraste, tenía pocas organizaciones de partido, como ya sabemos, puesto que allí la competencia anterior a la guerra había sido reducida a una sola cuestión: apoyar o no la secesión. Que la competencia entre Republicanos y Demócratas en el Norte sobrevivió a la guerra lo confirma el

${ }^{5}$ McKritick, sin embargo, reconoce que las raíces de este argumento han de buscarse en Poter (1960). 
hecho de que aunque los Republicanos controlaron todos los gobiernos estatales del norte tras la secesión, no consiguieron mantener el control en todas partes durante la guerra, del mismo modo que no fueron capaces de mantener el control de todos los parlamentos estatales. Los candidatos políticos republicanos, por tanto, se basaron a lo largo de la guerra en el liderazgo nacional de Lincoln para su supervivencia, mientras que Lincoln, que no estaba seguro de ganar a McClelland en las elecciones presidenciales de 1864, nunca perdió de vista la necesidad de mantener el apoyo de las organizaciones locales y estatales del partido Republicano. Por el contrario, ni Davis ni los gobernadores de los Estados de la Confederación hicieron frente a tales desafios. Con un partido republicano desacreditado, los gobernadores de los Estados sureños se enfrentaron a pocos desafíos inmediatos a su posición política, y poco era lo que tenían que ganar políticamente coordinándose con Davis.

La experiencia de Lincoln se ha repetido desde entonces. Ningún candidato a la presidencia de los EEUU puede esperar ganar sin el apoyo activo de los componentes estatales y locales de su partido, y cada cargo público estatal y local teme emprender la carrera hacia el cargo con un candidato presidencial débil a la cabeza de su partido. No obstante, si nos centramos en los EEUU sólo se pueden sugerir hipótesis, ya que, como destacamos anteriormente, muchos argumentarían que sus instituciones y tradiciones son únicas y no pueden ser copiadas. Por ello, es provechoso comparar los EEUU con otras federaciones, y para ese propósito consideraremos Alemania y Canadá, ya que una es una federación integrada y claramente estable, y la otra está menos integrada y es menos estable, al menos mientras que la cuestión de Quebec siga sin resolverse.

Claramente, lo que diferencia de manera más evidente el sistema político de Canadá y el de Alemania del norteamericano es el hecho de que son sistemas parlamentarios en lugar de presidenciales. Sin embargo, antes de centrarnos en esta distinción, comenzamos nuestra comparación observando que al menos un hecho sugiere que los EEUU es una federación mucho menos integrada que Canadá, y que Alemania, a pesar de su estructura parlamentaria, es más semejante a EEUU que Canadá. En concreto, aunque aproximadamente la mitad de los que consiguieron un cargo electo antes de ser presidentes de los EEUU fueron gobernadores de un Estado, ningún primer ministro de Canadá ha sido jefe de un gobierno provincial. De este modo, mientras que Schlessinger escribe de los políticos norteamericanos que "en los Estados, los hombres más vigorosos y ambiciosos son atraídos inexorablemente hacia la arena política nacional, bien al Senado o a algún lugar del complejo presidencial", Gibbins observa que en Canadá "hay muy poco movimiento de los cargos políticos provinciales hacia los cargos políticos nacionales. Los políticos aspirantes parece que hacen una elección temprana entre una carrera provincial o una carrera nacional, y una vez emprendido el camino pocas veces hay marcha atrás. El cargo provincial no es una estación en el camino hacia el cargo nacional, sino más bien una alternativa" 
(Schlessinger, 1966 y Gibbins, 1982). Lo que es especialmente interesante en nuestra triple comparación es que Alemania, a pesar de las diferencias aparentemente significativas en sus disposiciones institucionales, es igual que los EEUU en términos de la carrera que siguen sus figuras políticas nacionales. Por ejemplo, Kiesinger y Kohl fueron ambos presidentes de un Land antes de su nombramiento como cancilleres, Brandt fue alcalde de Berlín, y Schmidt comenzó su carrera política en la política local de Hamburgo. Que las federaciones de EEUU y Alemania están, al menos en términos del sistema de partidos, más integradas que la de Canadá, está confirmado por algunos otros hechos, incluyendo:

- Aunque en Canadá los Conservadores y los Liberales compiten en el ámbito nacional, a diferencia de los EEUU y Alemania, una diversidad de partidos políticos regionales o simplemente provinciales compiten unos con otros y con los dos partidos nacionales para cargos provinciales. En Alemania, como en EEUU," uno de sus tres partidos nacionales principales (generalmente la CDU/CSU o el SPD solos o en coalición con el FDP) controla cada uno de los gobiernos de los Länder.

- En EEUU la selección de delegados para las convenciones nacionales del partido Demócrata y Republicano está dominada por las organizaciones estatales del partido, que a su vez dependen en gran medida de sus organizaciones locales. De modo similar, en Alemania las convenciones nacionales del partido están dominadas por las organizaciones del partido en los Länder. Pero en Canadá, las convenciones nacionales están dominadas por las organizaciones nacionales del partido, que incluso a nivel provincial son generalmente distintas de las organizaciones que, con la misma etiqueta, sirven de candidatura a los políticos provinciales.

Tanto el sistema alemán de partidos como el americano, pues, son compatibles con una caracterización de federalismo que requiere que los sujetos federales sean una parte integrante del gobierno nacional, mientras que el sistema de partidos de Canadá está más en armonía con la caracterización de las relaciones federales como de $N+1$ participantes. Además, los políticos provinciales canadienses son elegidos normalmente en unas candidaturas que se presentan de forma explícita en oposición al gobierno nacional, y los votantes apuestan comúnmente por los políticos provinciales más capacitados para actuar como agentes de sus electores regionales, con el gobierno nacional identificado como fuerza hostil que debe ser controlada o moderada por ellos (Erikson y Filipov, 1996). Las disputas políticas entre niveles de gobierno son resueltas, si es que lo son, por una serie de pactos, a menudo contenciosos y largos, que son apoyados sólo hasta que surge la siguiente oportunidad de renegociación. Esto no quiere decir, por supuesto, que el regionalismo no entre en la mente de los votantes en EEUU o Alemania, o que los gobernadores de Estados o los jefes de los gobiernos regionales no hagan campaña de vez en cuando en oposición a la política federal. Pero lo más frecuente es que la competencia sea puramente partidista: un gobernador demócrata frente a un presidente republicano, o un miembro del SPD alemán frente a las políticas de 


\begin{tabular}{rr} 
R IS \\
EL DISEÑO DE UN ESTADO DEMOCRÁtICO FEDERAL VIABLE \\
\hline
\end{tabular}

una coalición gobernante diferente en Berlín. Pero en Canadá, la competencia es igual de probable entre políticos del mismo partido que entre políticos de distinto partido.

\section{DISEÑOS INSTITUCIONALES}

Si Alemania y EEUU son federaciones integradas y Canadá no, ¿a qué se debe la diferencia? No podemos, por supuesto, ofrecer una respuesta definitiva a tal cuestión, basada en tres estudios de caso, pero podemos intentar responder a nuestra pregunta con alguna especulación razonada. Primero, con respecto a Canadá y EEUU, hemos de observar que, a excepción de elecciones especiales, las elecciones locales, regionales y nacionales se producen en EEUU simultáneamente, mientras que en Canadá no siguen un calendario específico, y por ello, al ser proclamadas independientemente unas de otras, generalmente se producen en momentos distintos. Por tanto, la oportunidad para los políticos locales o regionales de ser elegidos como candidatos nacionales es mínima. Segundo, aunque en EEUU hay 600.000 cargos electos, los canadienses eligen sólo a sus representantes en el parlamento nacional y en el parlamento provincial, a los alcaldes y concejales, y a los consejos escolares (Gibbins, 1982: 112). Este hecho, combinado con el primero, implica que un votante americano cuando entra a la cabina de votaciones se enfrenta a una enorme lista de candidatos sobre los cuales se tiene poca $o$ ninguna información, salvo la información procedente de las etiquetas partidistas, mientras que un votante canadiense se enfrenta a un menú muy simple. Por tanto, es más probable que las etiquetas de los partidos nacionales sean mucho más útiles para encauzar la decisión de los votantes en las elecciones locales y regionales en EEUU que en Canadá. Igualmente, en mayor medida que sus homólogos canadienses, los políticos locales y regionales en EEUU tienen un fuerte incentivo para coordinar sus actividades electorales y para dar significado a esas etiquetas mediante el desarrollo de un sistema de partidos integrado.

Hay otras diferencias cruciales entre Canadá y EEUU, incluyendo el hecho de que el Senado de los EEUU, pero no el de Canadá, es un importante cuerpo legislativo que representa a los Estados explícitamente. Pero ahora queremos centrarnos en una segunda diferencia más sutil referida a los poderes del Presidente frente a los del Primer Ministro. Concretamente, aunque la estructura de las elecciones en EEUU fomenta un sistema de partidos integrado, de manera que ningún candidato a cargos nacionales, regionales o locales sería tan simple como para ignorarlo, este hecho no es necesariamente un motivo suficiente como para que un presidente o un gobernador dependa del partido cuando está gobernando. Este motivo deriva de un segundo hecho: la debilidad constitucional del cargo de presidente de EEUU (así como la debilidad equivalente de la mayoría de los gobernadores de los Estados). 
RIS

REVISTA INTERNACIONAL DE SOCIOLOGIA

No 32, Mayo-Agosto, 2002

PETER C. ORDESHOOK

A pesar del hecho de que EEUU es quizás el ejemplo más citado de sistema presidencial fuerte, una cuidadosa lectura de la Constitución de EEUU revela que ese cargo, al menos en términos de sus poderes formales, es débil: un presidente no puede firmar tratados o hacer un nombramiento sin el consentimiento del Senado; no desempeña papel alguno en lo que se refiere a las enmiendas de la Constitución; aunque sea comandante en jefe no puede declarar la guerra; no tiene autoridad especial en situaciones de emergencia; no tiene una fuente independiente de ingresos; no tiene autoridad sobre los gobiernos locales o estatales; no ocupa ningún cargo oficial en ningún partido político, y no tiene autoridad ejecutiva salvo la que le otorga el parlamento. Desde casi todos los puntos de vista, pues, tiene mucho menos poder formal que el de su homólogo Primer Ministro en un sistema parlamentario que, como cabeza de una coalición gobernante o un partido mayoritario, tiene mayor autoridad para llevar a cabo la legislación cotidiana y la administración del gobierno nacional y que, por definición, es el jefe de su partido. ¿Cuál es, entonces, la fuente de poder del Presidente de los EEUU y cómo influye en la integración y funcionamiento de un Estado federal?

La respuesta a esta pregunta comienza por observar que la autoridad política de un presidente de los EEUU deriva de un complejo equilibrio de rasgos institucionales. Como ilustra nuestra discusión sobre Lincoln y la Guerra Civil, ese poder deriva en gran medida del hecho de que el Presidente es la única figura elegida de ámbito nacional, lo cual, cuando se combina con las características previamente citadas de las elecciones norteamericanas, hace de ese cargo una fuerza política potencialmente muy integradora: un punto focal para el debate político. Pero considérese lo que sucedería si ese cargo estuviese dotado, además, de una gran autoridad formal como la que posee, por ejemplo, el Presidente de la Federación Rusa ${ }^{6}$. En ese caso nos imaginamos que pocos presidentes podrían resistirse a la tentación de gobernar mediante el ejercicio directo de su autoridad: promulgando decretos, amenazando con echar de sus cargos a los líderes políticos regionales y asignando fondos públicos estratégicamente entre amigos y enemi-

\footnotetext{
"Además de ser ungido como "garante de la Constitución rusa", entre los poderes constitucionales que posee el Presidente de la Federación Rusa y que no comparte con el Presidente de los EEUU se incluyen la autoridad para suspender las actividades administrativas de los gobiernos regionales que el presidente considere inconstitucionales (Artículo 85.2) y la autoridad para poner en marcha decretos ley siempre que no entren en conflicto con las leyes federales existentes (Artículo 90). Además, la Constitución de la Federación Rusa, en palabras que se remontan a la vieja noción de Lenin del centralismo democrático, proclama que "los organismos ejecutivos federales y los organismos con autoridad ejecutiva de los miembros de la Federación Rusa formarán un sistema único de autoridad ejecutiva" (Artículo 77.2).
} 
gos. Un presidente de los EEUU, sin embargo, no dispone de esos poderes, así que debe gobernar de manera diferente. Debe gobernar ejerciendo liderazgo. $Y$ aunque el significado de esa palabra es vago, incluye, en palabras de Theodore Roosevelt, el hábil ejercicio del "poder que te da hablar desde un púlpito".

Nuestro argumento, pues, es que el éxito del federalismo de los EEUU tiene dos fuentes interrelacionadas: la estructura de las elecciones y el establecimiento de un jefe del ejecutivo constitucionalmente débil, incluyendo un sistema ejecutivo federal - estatal descentralizado. La estructura de las elecciones hace de los partidos políticos el elemento potencialmente integrador fundamental del Estado, mientras que la descentralización de la autoridad ejecutiva y un presidente constitucionalmente débil exige que, para gobernar, el Presidente (así como los gobernadores de los Estados) use en la mayor medida posible el potencial integrador del partido, un potencial que sólo se puede llevar a la práctica mediante el uso de lo que llamamos liderazgo, más que a través del ejercicio directo y explícito del poder constitucional.

Obsérvese ahora que una importante implicación de este argumento es que ninguna estipulación constitucional puede ser considerada aisladamente. La medida en la cual una federación está o no integrada no depende de ninguna disposición constitucional única o un subapartado de la Constitución nacional. El carácter de una federación depende en cambio de la forma en que interactúa una amplia variedad de disposiciones constitucionales, incluyendo la estructura de las elecciones federales y regionales y la representación en el parlamento nacional, la autoridad ejecutiva del gobierno federal con relación a los gobiernos regionales y la autoridad legislativa del jefe del ejecutivo. Tratar de discernir o influir en el carácter de una federación centrándose sólo en aquellas estipulaciones constitucionales tradicional y explícitamente calificadas como "federales", sólo accidentalmente puede producir un federalismo integrado, y sospechamos que es más probable que produzca un diseño federal inapropiado.

Hay que reconocer que este argumento se deriva en gran medida de nuestra compresión del sistema político de los EEUU. Sin embargo, recibe un apoyo adicional si miramos a lo que se puede considerar como un federalismo igualmente exitoso, el de Alemania. A primera vista, Alemania parece institucionalmente más cerca de Canadá que de los EEUU. Es cierto que además de tener en común su forma parlamentaria, las elecciones a los Länder alemanes no se realizan necesariamente al mismo tiempo que las nacionales, muchos más cargos provinciales son cubiertos por nombramiento directo que mediante elecciones, y la cámara legislativa suprema de Alemania, el Bundesrat, no se aproxima de ninguna manera a la autoridad legislativa del Senado de los EEUU. Pero en compensación hay elementos que acercan el sistema alemán al modelo norteamericano. En primer lugar, la mitad de los escaños del Bundestag ocupados por listas de partidos es elegida usando las listas regionales (Länder). Así, los Länder tienen representación explícita en ambas cámaras legislativas e, igual- 
mente, en cualquier gobierno. En segundo lugar, esas listas, a diferencia de las nominaciones nacionales de los partidos en Canadá, son controladas por los mismos partidos regionales que eligen candidatos a cargos en los Länder. De este modo, los partidos que controlan el gobierno nacional son necesariamente entidades descentralizadas (federalizadas). En tercer lugar, aunque Alemania es parlamentaria, la autoridad constitucional que se le otorga a los Länder para la administración de las leyes y las políticas nacionales, da a los gobiernos de los Länder una voz directa en la rama ejecutiva del gobierno nacional y descentraliza la autoridad ejecutiva del Estado. Este último hecho también crea un Canciller alemán mucho más semejante a un presidente de los EEUU que a un primer ministro de Canadá: el asegurar la implantación ejecutiva de las leyes y programas federales requiere liderazgo, generalmente expresado a través de un sistema de partidos descentralizado pero integrado, más que el ejercicio directo del poder constitucional. Así, aunque la comparación de Alemania y EEUU revela que no hay ningún acuerdo institucional que garantice el desarrollo de una federación integrada, los acuerdos buscarían satisfacer los mismos principios del diseño federal para fomentar una relación simbiótica entre élites políticas en todos los niveles y divisiones del gobierno.

\section{UN ESTUDIO DE CASO: RUSIA}

Ya sea por casualidad o por un diseño inteligente, el sistema político alemán logra muchas de las mismas cosas que el de los EEUU, aunque cuenten con diferentes acuerdos institucionales debido a las diferencias existentes entre sus estructuras constitucionales fundamentales. La cuestión es si resulta posible equiparar otro experimento importante de diseño federal —el de la Federación Rusa - con las experiencias alemana y americana, si ha encontrado su propio camino hacia unas relaciones federales estables o si puede que esté imitando el ejemplo de tantas federaciones fallidas. Brevemente, nuestro argumento es que si subdividimos artificialmente la discusión anterior sobre las estipulaciones institucionales para centrarnos en aquéllas cosas que influyen más directamente en el desarrollo de partidos integrados y en aquéllas que requieren que la autoridad ejecutiva del Estado opere de una manera descentralizada, Rusia se acerca más a los requerimientos de una federación integrada sólo cuando se considera el segundo conjunto de estipulaciones. Pero en ausencia de una configuración institucional enteramente consistente, si nuestros argumentos anteriores son correctos, hay poca esperanza de que se pueda desarrollar completamente una federación integrada ${ }^{7}$. Las disposicio-

\footnotetext{
${ }^{7}$ Para una versión más elaborada del argumento que estamos ofreciendo en esta sección véase Ordeshook (1996); Ordeshook y Svetsova (1996).
} 
nes electorales parlamentarias de Rusia a este aspecto no son nada propicias para el desarrollo de partidos integrados, y su diseño ejecutivo, aunque sea adecuado para el mantenimiento de las relaciones federales en la rama ejecutiva, exacerba las imperfecciones del diseño parlamentario.

Aunque aparentemente modelado a imagen del sistema alemán, el mecanismo de formación de la cámara baja de Rusia, la Duma, no fomenta la creación de partidos políticos con bases regionales que estén integrados en alguna organización nacional. A diferencia de Alemania, el sistema ruso separa los destinos de los candidatos de las listas del partido de aquéllos que compiten en circunscripciones territoriales uninominales, y libera a los políticos que pertenecen a una organización nacional de tenerse que asegurar algo más específico que un voto nacional. Por otra parte, con 225 escaños de la Duma asignados a una circunscripción proporcional única que se extiende a lo largo de nueve zonas horarias a la vez y con un umbral del cinco por ciento para obtener representación, movilizar a los votantes dentro de cada una de las 89 regiones de Rusia es posible que resulte mucho más costoso que recurrir a esa circunscripción tan desperdigada usando un medio de comunicación nacional que funcione en gran medida fuera de Moscú. Con un electorado no regionalmente definido y dado que los votos en cualquier región concreta (salvo posiblemente Moscú y San Petersburgo) es poco probable que sean decisivos para superar el umbral, hay poca necesidad de que las élites del partido radicadas en Moscú ofrezcan a los políticos regionalmente populares un lugar en sus listas electorales nacionales lo suficientemente alto como para convertirlos en parte integral del esfuerzo de cualquier campaña electoral nacional.

Los distritos uninominales añaden pocos estímulos al desarrollo regional de los partidos. Al haber pocos cargos sometidos a elección competida aparte de éstos, las elecciones a la Duma en circunscripciones uninominales se centran en la personalidad de los candidatos antes que en las etiquetas de los partidos. De hecho, pocos candidatos acuden a la carrera electoral con una identificación partidista que no sea la etiqueta de "independiente", lo cual refleja los sentimientos anti-Moscú de muchos votantes y es un indicador seguro del carácter contencioso de las relaciones federales. Los candidatos que no son muy reconocidos por su nombre podrían atraer algún apoyo con la etiqueta del partido, pero no les interesa realizar su campaña apoyándose en un partido, ya que, en un contexto de negociación conflictiva, los partidos nacionales pueden ayudar poco al candidato local.

Por supuesto, uno podría argumentar que los partidos radicados en Moscú, al final construirán bases regionales de apoyo a medida que las instituciones políticas regionales se desarrollen y maduren. Sin embargo, es probable que esos incentivos sean superados por los incentivos asociados al uso del aparato administrativo del Estado para conducir las campañas nacionales. Con un ejecutivo nacional que puede suplantar muchas de las prerrogativas presupuestarias del parlamento y que puede ejercer su poder directamente sobre las administracio- 
RIS

nes regionales, con una Comisión Electoral Central en Moscú autorizada para regular el desarrollo de todas las elecciones, y con ejecutivos regionales que parecen diferenciarse poco de señores feudales, los gobernantes tiene incentivos irresistibles para corromper el proceso electoral.

Hay que admitir que si no se nos exigiera que nos preocupáramos de la viabilidad del proceso democrático o si estuviésemos seguros del desarrollo gradual de un sistema integrado de partidos, podríamos encontrar algún mérito en el diseño del poder ejecutivo en Rusia. De alguna forma se da una relación simbiótica entre los ejecutivos regionales y nacionales. Aunque incluso los gobernadores regionales tienen que ser elegidos directamente, las prerrogativas constitucionales del Presidente hacen que los gobernadores y los presidentes de las repúblicas dependan del gobierno nacional en aquellas cosas que éste facilita al gobierno regional (por ejemplo, acceso a la propiedad federal, reducción de impuestos, un puesto en la mesa de negociaciones de comercio internacional, y cerrar los ojos ante la corrupción local). Al mismo tiempo, los gobernadores elegidos popularmente, que el ejecutivo federal no puede pasar por alto, crean centros alternativos de poder y popularidad personales, y si se les pasa por alto, pueden hacer que toda perspectiva de apoyo electoral dentro de sus regiones se desvanezca. Porque el sistema electoral es muy fácilmente corruptible, y puesto que todo paso que se da para eliminar el fraude en las votaciones se centra en las maniobras burocráticas y es improbable que tenga un impacto global, los candidatos para el ejecutivo nacional dependerán en un futuro previsible de gobernadores regionales para tener "apoyo" en cualquier campaña electoral. Además, esta relación simbiótica, como en el sistema alemán, está fomentada por el evidente impulso hacia un modo descentralizado de ejecución de la ley federal, que sólo puede moderar la autoridad del ejecutivo nacional y alentar un uso mayor del "liderazgo" presidencial antes que de los poderes constitucionalmente regulados. Finalmente, esta simbiosis está fomentada además por un conflicto "natural" en el parlamento nacional entre la cámara baja, populista, y la cámara alta, que está controlada en gran medida por ejecutivos regionales. El subsiguiente triángulo de poder formado por la Duma, el Consejo de la Federación y la Presidencia abre las puertas a un sistema equilibrado de poder federal que, para evitar callejones sin salida, puede promover que las élites políticas en todas sus ramas (tanto regionales como nacionales) se basen en los partidos como mecanismo principal de coordinación.

Sin embargo, las deficiencias de la estructura política institucional en Rusia probablemente impidan la realización de una federación integrada. En primer lugar, aparte de los fallos anteriormente observados en el sistema electoral nacional, está el hecho de que el Presidente no sólo posee poderes ejecutivos fuertes, sino que tiene también amplios poderes legislativos y judiciales (véanse especialmente los artículos 85 y 90 de la Constitución de la Federación Rusa). Así, aunque un presidente puede ser alentado a gobernar a través del liderazgo con respecto a las 
administraciones regionales, sus relaciones con las otras ramas del gobierno estarán basadas, con mayor probabilidad, simplemente en la autoridad constitucional de ese cargo, que sólo puede ahogar el desarrollo de un sistema integrado de partidos políticos. En segundo lugar está el hecho de que las elecciones, incluso para cargos puramente regionales, no se realizan simultáneamente. Así, al aislar las elecciones de los gobernadores de todas las demás, Rusia fomenta campañas puramente personalistas, o campañas en las cuales, como en Canadá, la cuestión más destacada es la oposición al gobierno nacional. Tercero, la relación simbiótica que existe entre los ejecutivos nacionales y regionales, en combinación con el carácter personalista de las elecciones a gobernador, es muy probable que fomente una forma de jefatura en la cual los parlamentos regionales permanezcan subordinados a los gobernadores regionales. En este aspecto, por lo tanto, el sistema político de Rusia comienza a parecerse más a una variante de la vieja forma de centralismo democrático de la URSS, que a una nueva variante de federalismo democrático.

Aún queda, por supuesto, la posibilidad de que Rusia modifique sus sistemas electorales y constitucionales para que fomente mejor la evolución hacia una federación integrada. Y siempre existe la posibilidad de que nuestros argumentos sean incompletos. Pero si son completos en gran medida y si no se intenta ningún ajuste institucional significativo, nos quedan pocas alternativas excepto predecir que las relaciones federales rusas no se parecerán a las de EEUU o las de Alemania. Antes bien, asumiendo que la Federación misma sobreviva en alguna forma democrática, se equipararán más probablemente, en un primer escenario, a las que actualmente se dan en Canadá, o se equipararán, en un segundo, a la configuración más unitaria de Estado pseudofederal que se da en México.

\section{BIBLIOGRAFÍA}

BUCHANAN, J.M. (1995), "Federalism as an ideal political order and an objective for Constitutional reform", Publius, 25.2, Invierno, pp.19-28.

BURGESS, M. y A.G. GAGNON (1993), Comparative Federalism and Federation, Toronto, University of Toronto Press.

CHANDLER, W.M. (1987), "Federalism and Political Parties", Federalism and the Role of the State, Toronto, University of Toronto Press.

EASTERLIN, R.A. (1960), "Interregional Differences in Per Capita Income, Population and Total Income, 1840-1950", en Trends in the American Economy in the Nineteenth Century, Informe NBER, vol. 24, Princeton, Princeton University Press.

ERIKSON, R.S. y M. G. FILIPOV (1996), "Ideological balancing in elections. The Case of Canada", ponencia presentada en el Congreso Anual de la Asociación Americana de Ciencia Politica, San Francisco. 
RIS

FRIEDMAN, J.W. (1977), Oligopoly and the Theory of Games, Amsterdam, North-Holland.

GIBBINS, R. (1982), Regionalism: Territorial Politics in Canada and the United States, Toronto, Butterworth and Co.

HOROWITZ, D. (1985), Ethnic Groups in Conflict, Berkeley, University of California Press.

(1991), A Democratic South Africa?, Berkeley, University of California Press.

LIJPHART, A. (1984), Democracies, New Haven, Yale University Press.

McKITRICK, E.L. (1967), "Party Politics and the Union and Confederate War Efforts", en William N. Chambers y Wlater D. Burnham (eds.), The American Party System, Nueva York, Oxford University Press.

ORDESHOOK, P.C. (1996), “Russia's party system: Is Russian federalism viable?”, Post-Soviet Affairs, vol. 12, n 3, pp. 195-217.

ORDESHOOK, P.C. y O. SVETSOVA (1996), "Federalism and parties: will russia be Mexico or Germany", ponencia presentada en el Congreso Anual de la Asociación Americana de Ciencia Política, San Francisco.

OSTROM, V. (1991), The Meaning of America Federalism, San Francisco, ICS Press.

POTER, D.M. (1960), “Jefferson Davis and the Political Factors in Confederate Defeat”, en David Donald (ed.), Why the North Won the Civil War, Baton Rouge.

RIKER, W.H. (1964), Federalism, Boston, Little Brown.

SAUNDERS, C. (1995), “Constitutional Arrangements of Federal Systems”, Publius, n² 25, Invierno, pp.61-80.

SCHLESSINGER, J. (1966), Ambition and Politics: Political Careers in the United States, Chicago, Randall MCNally.

SMITH, G. (1995), Federalism: the Multiethnic Challenge, London, Longman.

TAYLOR, M. (1976), Anarchy and Cooperation, Nueva York, Wiley.

TUSHNET, M. (1990), Copararative Constitutional Federalism, Greenwood.

WALLICH, C. (1994), Russia and the Challenges of Fiscal Federalism, Washington, D.C., World Bank. 